\title{
Disturbed Anterior Prefrontal Control of the Mesolimbic Reward System and Increased Impulsivity in Bipolar Disorder
}

\author{
Sarah Trost*,', Esther Kristina Diekhof ',2, Kerstin Zvonik', Mirjana Lewandowski', Juliana Usher', \\ Maria Keil', David Zilles', Peter Falkai ${ }^{3}$, Peter Dechent ${ }^{4}$ and Oliver Gruber' \\ 'Department of Psychiatry and Psychotherapy, Centre for Translational Research in Systems Neuroscience and Clinical Psychiatry, Georg August \\ University, Goettingen, Germany; ${ }^{2}$ Biocenter Grindeland Zoological Museum, Institute for Human Biology, University of Hamburg, Hamburg, \\ Germany; ${ }^{3}$ Department of Psychiatry and Psychotherapy, Ludwig-Maximilians-University, Munich, Germany; ${ }^{4}$ Department of Cognitive \\ Neurology, Georg August University, Goettingen, Germany
}

Bipolar disorder $(\mathrm{BD})$ is characterized by recurrent mood episodes ranging from severe depression to acute full-blown mania. Both states of this severe psychiatric disorder have been associated with alterations of reward processing in the brain. Here, we present results of a functional magnetic resonance imaging ( $\mathrm{MRR}$ ) study on the neural correlates and functional interactions underlying reward gain processing and reward dismissal in favor of a long-term goal in bipolar patients. Sixteen medicated patients diagnosed with bipolar I disorder, euthymic to mildly depressed, and sixteen matched healthy controls performed the 'desire-reason dilemma' (DRD) paradigm demanding rejection of priorly conditioned reward stimuli to successfully pursue a superordinate goal. Both groups exhibited significant activations in reward-related brain regions, particularly in the mesolimbic reward system. However, bipolar patients showed reduced neural responses of the ventral striatum (vStr) when exploiting a reward stimulus, and exhibited a decreased suppression of the rewardrelated activation of the mesolimbic reward system while having to reject immediate reward in favor of the long-term goal. Further, functional interaction between the anteroventral prefrontal cortex and the vStr in the 'DRD' was significantly impaired in the bipolar group. These findings provide evidence for a reduced responsivity of the vStr to reward stimuli in BD, possibly related to clinical features like anhedonia. The disturbed top-down control of mesolimbic reward signals by prefrontal brain regions in BD can be interpreted in terms of a disease-related enhanced impulsivity, a trait marker of BD.

Neuropsychopharmacology (2014) 39, 1914-1923; doi:I0.1038/npp.2014.39; published online 19 March 2014

\section{INTRODUCTION}

Bipolar disorder (BD) is a severe psychiatric illness characterized by recurrent mood episodes ranging from severe depression to acute full-blown mania. Both depressive and manic mood states have been associated with dysfunctions of the brain reward system (Alloy et al, 2012; Hasler, 2006; Johnson et al, 2012b). Previous studies have shown that bipolar patients exhibit increased behavioral and emotional responses to reward or its anticipation compared with healthy controls (Alloy et al, 2012; Gruber, 2011; Johnson et al, 2012a, 2012b). However, bipolar patients do not appear to be faster to learn stimulus-reward associations (Johnson et al, 2012b; O'Sullivan et al, 2011) and even exhibit deficits in probabilistic reversal learning compared with healthy controls (Dickstein et al, 2010;

*Correspondence: Dr S Trost, Department of Psychiatry and Psychotherapy, Centre for Translational Research in Systems Neuroscience and Clinical Psychiatry, Georg August University, Goettingen 37075, Germany, Tel: +49 551 39 101 15/6615 (-8952), Fax: +49 551 398952, E-mail: s.trost@med.uni-goettingen.de

Received II October 2013; revised 12 February 2014; accepted 12 February 2014; accepted article preview online 18 February 2014
Gorrindo et al, 2005). It has been shown that once bipolar patients have associated a stimulus with reward, they are slow to realize changes in contingencies and still continue to choose a previously rewarded response (Johnson et al, 2012b).

Reward processing in the human brain is based on a neuronal circuitry including regions of the mesolimbic dopamine system, in particular the ventral striatum (vStr) and ventral tegmental area (VTA) (Diekhof and Gruber, 2010; Diekhof et al, 2008; Liu et al, 2011). Intriguingly, gray matter deficits in vStr and mesial prefrontal cortex have been associated with genetic risk for $\mathrm{BD}$ in a volumetric magnetic resonance imaging (MRI) study (McDonald et al, 2004). So far, only few functional magnetic MRI (fMRI) studies on reward processing in BD have been implemented. Some of these studies indicate atypical activation effects in different parts of the reward circuitry, especially during hypomania and mania (Abler et al, 2008; Bermpohl et al, 2010; O'Sullivan et al, 2011). During euthymia, Nusslock et al (2012) reported elevated ventral striatal and orbitofrontal activity during reward anticipation, but not outcome, in bipolar I patients, while Caseras et al (2013) found elevated ventral striatal activity in bipolar II patients rather 
than in bipolar I patients. A study by Linke et al (2012) using a probabilistic reversal learning task showed increased activity of the orbitofrontal cortex in response to reward and an attenuated prediction error signal in euthymic bipolar patients.

A recent fMRI study by Diekhof and Gruber (2010) assessed the neural correlates of reward processing as well as functional interactions between brain areas when actions promoting a superordinate goal precluded exploitation of an immediately available reward option. Here, we used a modified version of this 'desire-reason dilemma' (DRD) paradigm (Diekhof and Gruber, 2010) to investigate the reward processing circuitry in bipolar patients compared with healthy controls. We hypothesized that bipolar patients would fail to consequently pursue a superordinate goal at the expense of immediate reward because of clinging to initially established stimulus-reward-contingencies and enhanced trait impulsivity. Furthermore, we presumed altered brain activation patterns in the reward circuitry and impaired top-down control mechanisms underlying these behavioral deficits in BD.

\section{MATERIALS AND METHODS}

\section{Subjects}

Twenty-four patients participated in the study. All patients met the diagnostic criteria for $\mathrm{BD}$, type 1 , according to ICD-10 and DSM-IV classification standards. Diagnoses were consented within members of the study group and the treating clinical psychiatrist. Three patients had to be excluded because of insufficient task performance, and another five patients due to poor quality of fMRI data (motion artifacts). Subsequently, 16 healthy control subjects out of 24 control subjects recruited for this patient study were matched to the remaining 16 patients according to age, gender, and education.

The healthy controls exhibited no past or present psychiatric or neurological disorder and had no positive family history of psychiatric disorders. Exclusion criteria in general were lifetime diagnoses of substance dependence, substance abuse during the last month, cannabis abuse during the last 2 weeks, mental retardation, dementia, and neurological illnesses.

Demographic, personality, and clinical data for the study sample are given in Table 1 . Seven out of sixteen patients were euthymic as defined by MADRS $<7$ points and YMRS $<7$ points. Eight patients presented with mild depressive symptoms scoring $\geqslant 10$ points on the MADRS with a maximum of 24 points in one patient. Two patients displayed mild mixed symptoms scoring on the YMRS with $\geqslant 10$ points. None of the patients included was suffering from a severe manic, depressive, or mixed episode.

None of the healthy controls or patients reported current substance abuse. Two patients included reported alcohol abuse in the past and one patient reported cannabis abuse in the past ( $>1$ year ago). None of the patients included had been hospitalized for alcohol or drug addiction treatment or had suffered from withdrawal symptoms. The control subjects reported no substance abuse (including cannabis) in the past in our questionnaires.
Written informed consent was obtained from all subjects before their inclusion into the investigation. The study was performed in accordance with the ethical standards laid down in the Declaration of Helsinki (Williams, 2008) and was approved by the local ethics committee. Subjects were paid for participation.

\section{Experimental Procedure}

First, participants underwent a training session outside the scanner. The training started with an operant conditioning task. Squares of eight different colors were presented on a monitor in a shuffled mode. Subjects were instructed to respond to each of the presented colored squares (stimulus) by button press (left button: accept; right button: reject). Button choice was free in the conditioning phase and subjects were encouraged to explore the stimulus-responsereward contingencies. By exploring the stimulus-responsereward contingencies, subjects were conditioned to associate two of the eight colors (red and green) with an immediate reward (bonus of +10 points), while the other colors were associated with a neutral outcome. The goal of this operant conditioning task was to establish stimulusresponse-reward contingencies for the next phase of the experiment.

Subsequently, subjects were familiarized with the actual reward task, the 'DRD' paradigm, a sequential forced-choice task (see Figure 1). Subjects had to pursue a superordinate long-term goal during task blocks of 4-7 trials to acquire 50 points at the end of each block. Two different types of blocks had to be performed. In the first type of blocks, the 'desire context' (DC), subjects were allowed to collect priorly conditioned reward stimuli and win additional points. In the second type of blocks, the 'reason context' (RC), the conditioned reward stimuli had to be rejected to successfully pursue the long-term goal (50 points at the end of the block of trials). So, during the RC, subjects were forced to overcome the tendency to acquire immediate reward to reach the superordinate long-term goal. This condition therefore constituted a DRD (Diekhof and Gruber, 2010; Diekhof et al, 2012b). For more information, see Supplementary Materials.

\section{Behavioral Data Analyses}

Statistical analyses of performance data were done using the software package SPSS for windows (IBM SPSS Statistics 19.0). T-tests were used to test for differences between groups (two-tailed significance). Normal distribution of performance data between groups was tested using the Kolmogorov-Smirnov test.

\section{fMRI Data Acquisition and Analyses}

fMRI was performed on a 3 Tesla scanner (Magnetom TIM Trio; Siemens Healthcare, Erlangen, Germany) equipped with the standard eight channel phased-array head coil. First, a T1-weighted anatomical data set with $1 \mathrm{~mm}$ isotropic resolution was acquired. For fMRI, 31 axial slices parallel to the anterior commissure-posterior commissure line were acquired in ascending direction (slice thickness = $3 \mathrm{~mm}$; interslice gap $=0.6 \mathrm{~mm}$ ) using a gradient-echo 
Table I Demographic, Personality, and Clinical Data of Study Subjects

\begin{tabular}{|c|c|c|c|}
\hline & Bipolar patients & Healthy controls & $p$-Value \\
\hline \multicolumn{4}{|l|}{ Demographic data } \\
\hline Gender & $6 \mathrm{M}, 10 \mathrm{~F}$ & $7 \mathrm{M}, 9 \mathrm{~F}$ & 0.762 \\
\hline Age (years) & $35.6 \pm 9.2$ & $35.4 \pm 9.9$ & 0.971 \\
\hline Range (years) & $23-49$ & $21-48$ & \\
\hline Education (years) & $14.9 \pm 3.3$ & $14.7 \pm 2.5$ & 0.854 \\
\hline \multicolumn{4}{|l|}{ Barratt Impulsiveness Scale scores (BIS-I I) } \\
\hline BIS-I I motor impulsiveness & $18.56 \pm 3.6$ & $16.63 \pm 3.0$ & 0.109 \\
\hline BIS-I I cognitive impulsiveness & $23.31 \pm 5.3$ & $21.94 \pm 2.7$ & 0.367 \\
\hline BIS-I I non-planning impulsiveness & $26.88 \pm 4.2$ & $23.38 \pm 3.9$ & $0.020^{\mathrm{a}}$ \\
\hline MADRS item 8 (inability to feel) & $1.0 \pm 1.5$ & & \\
\hline CGl & $3.7 \pm 1.0$ & & \\
\hline YMRS & $2.3 \pm 5.6$ & & \\
\hline \multicolumn{4}{|l|}{ Clinical data } \\
\hline Age of onset (years) & $24.5 \pm 6.8$ & & \\
\hline Polarity of first episode & Manic $n=10$, depressive $n=6$ & & \\
\hline Duration of illness (years) & $11.1 \pm 7.2$ & & \\
\hline Number of episodes & $5.9 \pm 3.6$ & & \\
\hline Number of manic episodes & $2.5 \pm 1.6$ & & \\
\hline \multicolumn{4}{|c|}{ Psychotropic medication at the time of the investigation } \\
\hline Antidepressants & $9 / 16$ & & \\
\hline Atypical antipsychotics & $8 / 16$ & & \\
\hline Benzodiazepines & $0 / 16$ & & \\
\hline Unmedicated patients & $2 / 16$ & & \\
\hline CPZ equivalents (daily) & $\mid 48.4 \pm 191.7$ & & \\
\hline
\end{tabular}

Abbreviations: CGI, Clinical Global Impression Score; CPZ, chlorpromazine equivalent dose; F, female; M, male; MADRS, Montgomery-Asberg Depression Rating Scale; n, number; YMRS, Young Mania Rating Scale.

Data are presented as mean \pm standard deviation unless otherwise indicated.

asignificant $p<0.05$.

echo-planar imaging sequence (echo time $33 \mathrm{~ms}$, flip angle $70^{\circ}$; field-of-view $192 \mathrm{~mm}$, interscan repetition time $1900 \mathrm{~ms})$.

In all, 185 volumes each were acquired in 2 functional runs. Stimuli were viewed through goggles (Resonance Technology, Nothridge, USA), and subjects responded via button presses on a fiber optic computer response device (Current Designs, Philadelphia, USA). Triggering of the visual stimulation by the scanner impulse during functional data acquisition and generation of stimuli was performed using the Presentation Software (Neurobehavioral Systems, Albany, USA).

Functional images were preprocessed and analyzed with SPM5 (Statistical Parametric Mapping; www.fil.ion.ucl. ac.uk/spm/software/spm5/) using a general linear model (see Supplementary Materials). The study design was eventrelated and only correctly answered trials were included in the analysis.

Linear $\mathrm{t}$-contrasts were defined to assess brain activation effects in the two contexts. We analyzed the activation 


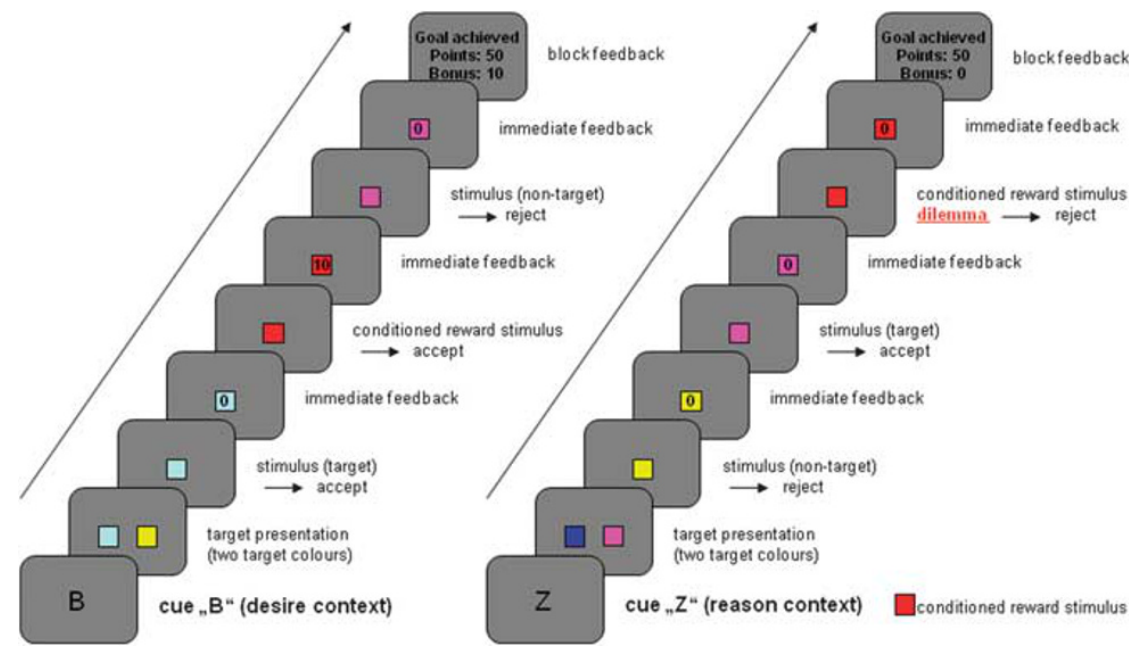

Figure I Experimental design of the 'desire-reason dilemma' paradigm. 'Desire context' (DC, left): participants had to accept the two target stimuli to achieve the superordinate goal, non-targets had to be rejected. Participants were free to additionally accept the priorly conditioned reward stimuli (red and green) and were rewarded with + 10 points each in case of acceptance. In the 'reason context' (RC, right) participants had to accept the two target stimuli and reject all other stimuli including the priorly conditioned reward stimuli to achieve the superordinate goal (50 points at the end of the block). In this dilemma situation, participants had to overcome the behavioral bias toward immediate reward to optimize their behavioral outcome.

effects elicited by the conditioned reward stimuli in the DC and compared these effects with the effects of the same conditioned stimuli when being presented in the RC. We contrasted DC with RC to assess the extent of downregulation (suppression) of reward-related activation during a competition between the superordinate goal and the proximal reward option in the RC.

Second-level analyses were performed using single subject contrast images to assess group effects. The statistical search criterion for group statistics was $p<0.005$, uncorrected, if not otherwise indicated.

To control for multiple testing, region of interest (ROI) analyses were performed around a priori coordinates from previous studies (Diekhof and Gruber, 2010; Diekhof et al, 2012a, 2012b) employing a significance threshold of $p<0.05$, FWE-corrected for small volume. Small volume correction was performed for the bilateral vStr and bilateral VTA ( vStr $\pm 1212-3$; $6 \mathrm{~mm}$ box, VTA $\pm 6-21-18$; $6 \mathrm{~mm}$ box, coordinates taken from Diekhof and Gruber (2010)) and the anteroventral prefrontal cortex (avPFC) (median from previous publications $\pm 30513 ; 6 \mathrm{~mm}$ box, taken from Diekhof and Gruber (Diekhof and Gruber, 2010; Diekhof et al, 2012a, 2012b); small volume correction done in psychophysiological interaction (PPI) contrast, see below).

For further analyses of ROI data, the MARSBAR (http:// marsbar.sourceforge.net) software was used to extract each ROI's mean BOLD parameter estimate value (beta value) for each participant. Mean beta values were extracted from coordinate-centered spheres around the bilateral vStr ( $3 \mathrm{~mm}$ radius; $\pm 1212-3$ ) and the avPFC (5 mm radius; mean coordinates from previous studies 3051 3), a priori ROIs from Diekhof and Gruber (Diekhof and Gruber, 2010; Diekhof et al, 2012a, 2012b). Post hoc, we extracted beta values (coordinate-centered sphere $5 \mathrm{~mm}$ radius) from all differentially activated brain regions between groups in the DC and the RC (see Tables 2 and 3; bipolar patients $<$ healthy controls). On an exploratory basis, Pearson correlation coefficients were calculated between beta values and clinical parameters, performance data, and BIS-11 scores.

\section{PPI Analyses}

We assessed the functional interactions of the vStr (nucleus accumbens) with prefrontal brain regions, especially the avPFC in the DRD situation using PPI analyses (Friston et al, 1997). Prior investigations of our group had shown negative functional interactions between the vStr and the avPFC in healthy subjects successfully performing the DRD (Diekhof and Gruber, 2010). We selected the bilateral vStr $\left(\begin{array}{lll} \pm 12 & 12 & -3\end{array}\right)$ as seed areas for the PPI. For more information, see Supplementary Materials.

\section{RESULTS}

\section{Behavioral Results}

Performance data were normally distributed according to the Kolmogorov-Smirnov test for two samples. Overall performance rates for correct acceptance of non-rewarded target stimuli and correct rejection of non-rewarded nontarget stimuli slightly differed between groups (bipolar patients: $84.5 \pm 9.8 \%$; healthy controls: $87.3 \pm 7.3 \%$ ), but this difference was not significant. However, the bipolar patients exhibited significantly lower mean percentage rates of correct acceptance of conditioned reward stimuli in the DC (bipolar patients: $72.2 \pm 17.7 \%$; healthy controls: $86.0 \pm 12.9 \%$; $p=0.029)$. Likewise in the RC, in which the subjects had to refrain from accepting the reward stimulus to pursue the superordinate task goal, patients performed significantly worse than the controls (bipolar patients: $79.7 \pm 22.3 \%$; healthy controls: $92.1 \pm 14.9 \% ; p=0.040$ ). Furthermore, the bipolar patients showed significantly lower percentage rates of correct target detection, interpretable as a reduced working memory capacity for color 
Table 2 Brain Regions Activated by the Conditioned Reward Stimuli in the 'Desire Context' (DC)

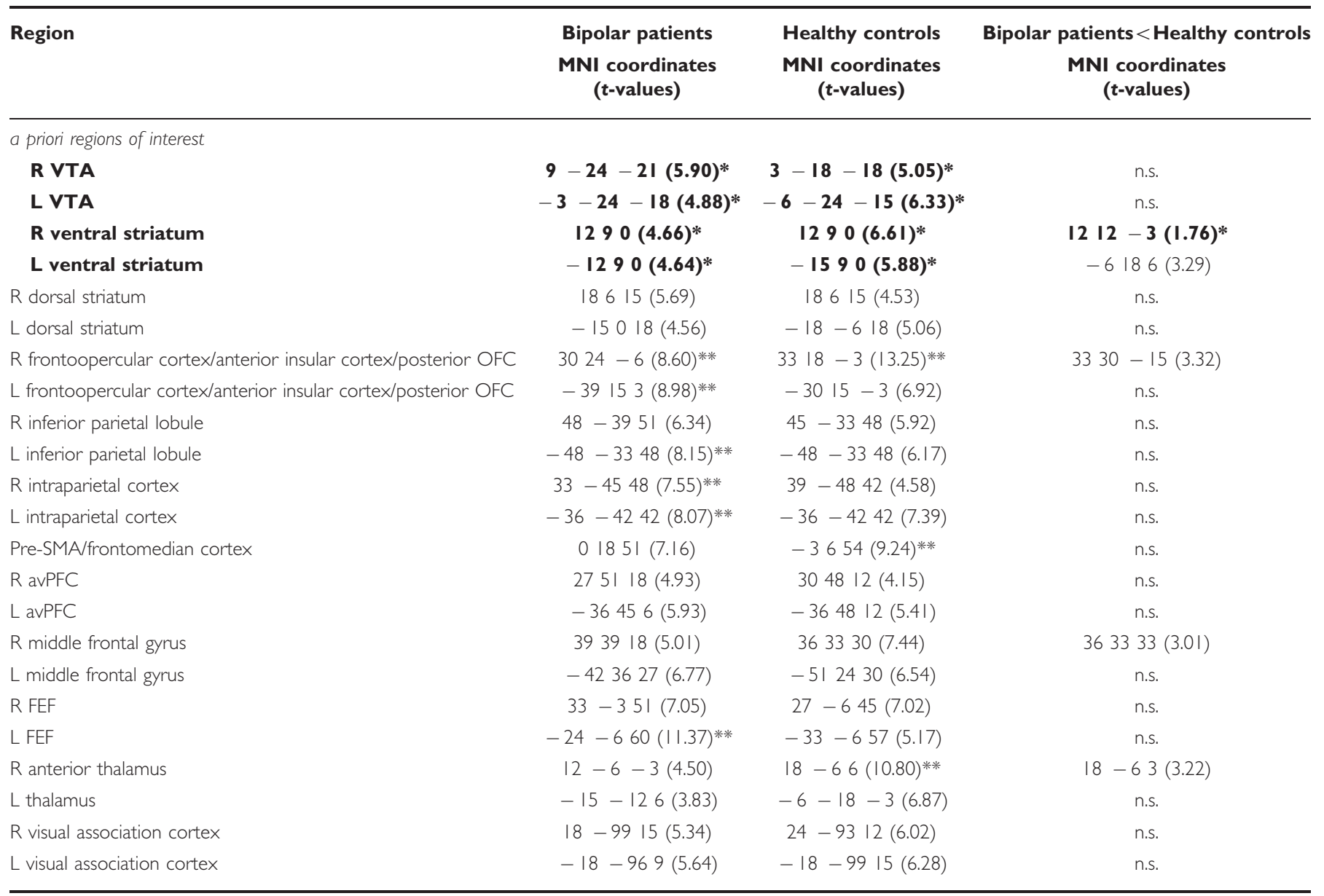

Abbreviations: avPFC, anteroventral prefrontal cortex; FEF frontal eye fields; L, left; n.s., not significant; OFC, orbitofrontal cortex; R, right; SMA, supplementary motor area; VTA, ventral tegmental area.

Effects on regional brain activation were searched for using the primary search criterion of $p<0.005$, uncorrected. $* p<0.05$, FWE-corrected for small volume ( 6 mm box) around a priori coordinates from Diekhof and Gruber (2010); ** $<0.05$, FWE-corrected for the entire brain.

Bold values indicate the regions and coordinates are the a priori regions of interest (bilateral VTA and ventral striatum), FWE-corrected coordinates are in bold to put emphasis on these regions and coordinates.

features (percentage of correctly accepted non-rewarded target stimuli; bipolar patients: $90.8 \pm 7.1 \%$; healthy controls: $95.8 \pm 2.8 \% ; p=0.026)$.

\section{Functional Magnetic Resonance Imaging}

In the DC, both bipolar patients and controls showed reward-related brain activations in subcortical structures, ie, in bilateral vStr and VTA/midbrain (Table 2). This is consistent with previous fMRI studies associating these brain structures with reward prediction and choice of immediate reward (Diekhof and Gruber, 2010; Knutson et al, 2001; McClure et al, 2004; O’Doherty, 2004). Furthermore, both groups exhibited activations in an extended bilateral frontoparietal network, including prefrontal, orbitofrontal, and parietal cortices (Table 2).

Bipolar patients showed significantly lower reward-related brain activation in the bilateral vStr $(p<0.05$, FWE-corrected for small volume), the right anterior thalamus, and several prefrontal cortical regions than the control group (Table 2). Inversely, the bipolar patients did not show more rewardrelated activation than the controls in any brain region.
Previous studies in healthy subjects had repeatedly shown downregulation of brain activation in the bilateral vStr during the successful performance in the DRD (Diekhof and Gruber, 2010; Diekhof et al, 2012a, 2012b). While both groups showed this downregulation of activation in the bilateral vStr as well as in an extended fronto-parietal cortical network, the extent of suppression was significantly reduced in the bipolar patients compared with the controls (Table 3$)$, in particular in the vStr $(p<0.05$, FWE-corrected for small volume). Conversely, there were no brain areas with significantly increased suppression effects in the patient group compared with healthy controls (Table 3).

\section{PPI Results}

Replicating our previous findings in the DRD paradigm (Diekhof and Gruber, 2010), we found a significantly increased negative functional interaction between the vStr and the right avPFC in the control group (PPI with seed $\mathrm{R}$ vStr: $33513 ; t=4.38 ; p<0.05$, FWE-corrected for small volume; PPI with seed L vStr: $335115 ; t=3.00 ; p \leqslant 0.05$, FWE-corrected for small volume). In contrast to that, the 
Table 3 Suppression of Reward-Related Activity due to the 'Desire-Reason Dilemma' (DRD)

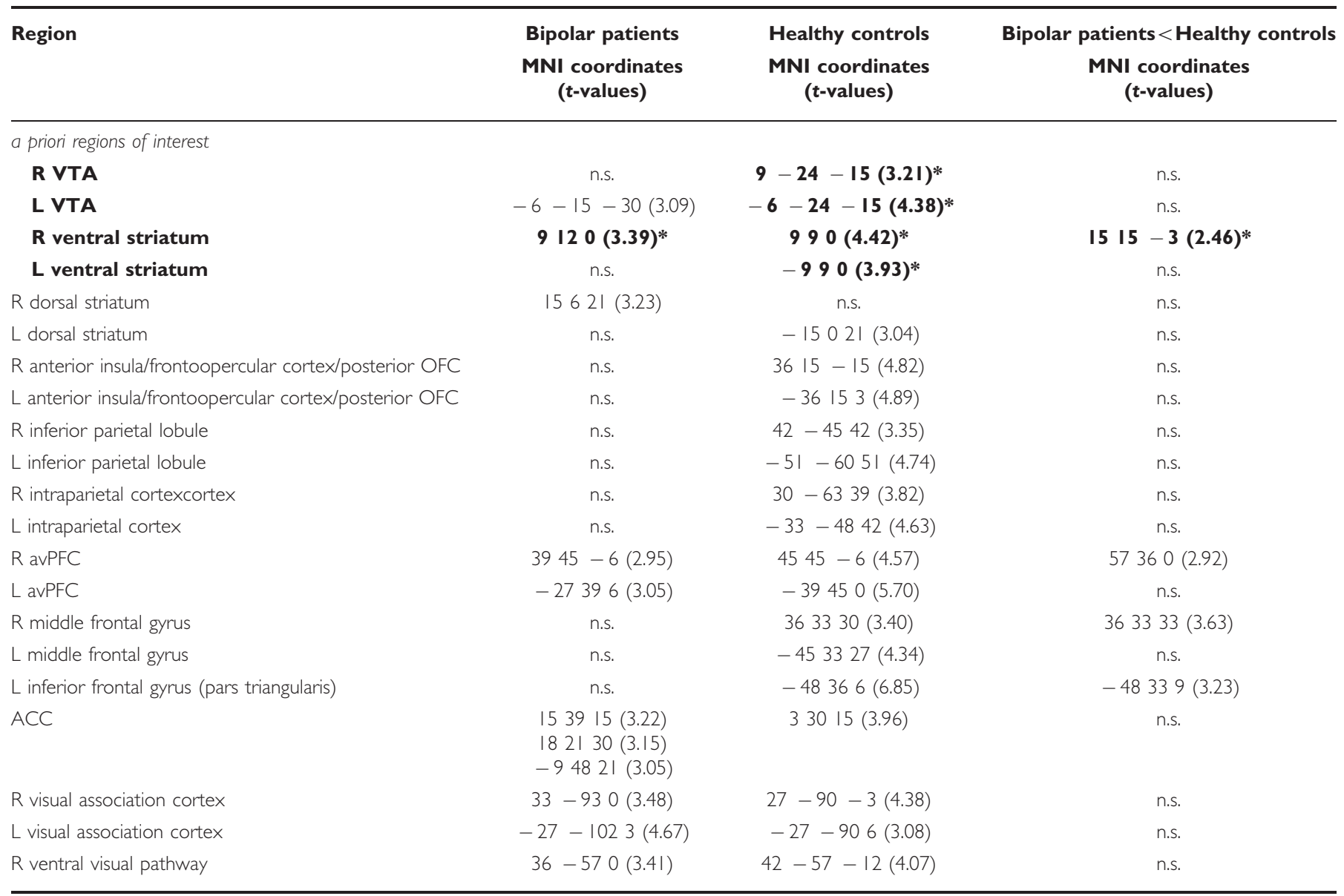

Abbreviations: ACC, anterior cingulate cortex; avPFC, anteroventral prefrontal cortex; L, left; n.s., not significant; OFC, orbitofrontal cortex; R, right; VTA, ventral tegmental area.

Statistical effects represent significant reductions of reward-induced activation in the reason context as compared with the desire context. Effects on regional brain activation were searched for using the primary search criterion of $p<0.005$, uncorrected. ${ }^{*} p<0.05$, FWE-corrected for small volume ( $6 \mathrm{~mm}$ box $)$ around a priori coordinates from Diekhof and Gruber (2010).

Bold values indicate the regions and coordinates are the a priori regions of interest (bilateral VTA and ventral striatum), FWE-corrected coordinates are in bold to put emphasis on these regions and coordinates.

bipolar patient group did not show this negative functional coupling, but only exhibited subthreshold positive functional interaction between bilateral vStr and the avPFC at a low level (PPI with seed R vStr: $42516 ; t=1.85 ; p<0.05$, uncorrected; PPI with seed L vStr: 3648 6; $t=1.88 ; p<0.05$, uncorrected). The group comparison confirmed a significantly reduced negative functional interaction of the right and left vStr with the avPFC (PPI with seed R vStr: 3948 3; $t=3.97 ; p<0.05$, FWE-corrected for small volume; PPI with seed L vStr: 3648 6; $t=2.77 ; p<0.05$, FWE-corrected for small volume) in the bipolar patients (Figure 2).

Beta values of the right avPFC extracted from the PPI analyses (seed R and L vStr) of each participant were not significantly correlated with clinical parameters, performance data or BIS-11 scores (see also below, exploratory correlational analyses).

\section{Exploratory Correlational Analyses}

There were no significant correlations between beta values of the bilateral vStr in DC or RC and MADRS,
MADRS item 8 (anhedonia), CGI, YMRS, performance data, or BIS-11 scores. But, we found a significant positive correlation between beta values indicating the extent of suppression of activity in the bilateral vStr in the RC compared with the DC and CPZ (chlorpromazine) equivalent doses in the bipolar patients (left vStr $p=0.029$; right vStr $p=0.045$ ). Furthermore, post hoc correlation analysis between beta values of the left inferior frontal gyrus (sphere $5 \mathrm{~mm}$ radius; center -48339 ; coordinates from group comparison bipolar patients $<$ healthy controls, see Table 3 ) in the RC and BIS-11 total scores showed a significant negative correlation in the bipolar patients $(p=0.044)$. Also post hoc, we found a significant positive correlation between beta values extracted from the right middle frontal gyrus in the DC (sphere $5 \mathrm{~mm}$ radius, center 3633 33; coordinates from group comparison bipolar patients $<$ healthy controls, see Table 2) and performance data (percentage of correctly accepted non-rewarded target stimuli) in bipolar patients and healthy controls $(p=0.017)$. No other significant correlation between beta values and clinical parameters, performance data or BIS-11 scores was found. 

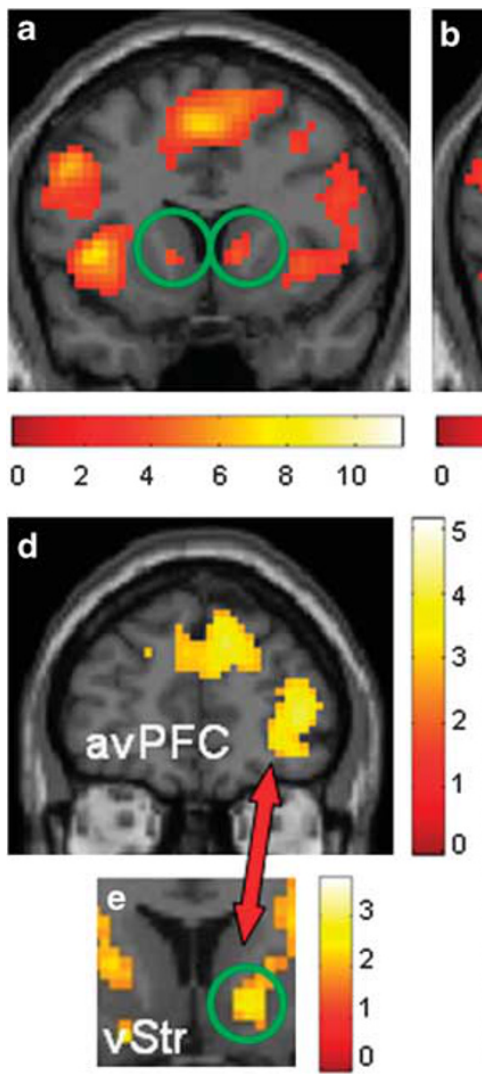
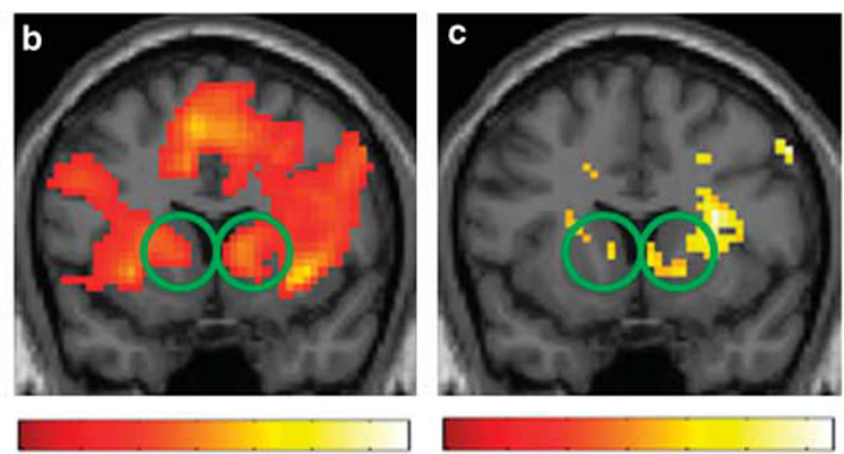

$\begin{array}{cccccccccccccc}0 & 2 & 4 & 6 & 8 & 10 & 12 & 0 & 0.5 & 1 & 1.5 & 2 & 2.5 & 3 \\ 5 & \text { A-C. Bottom-up activation of the } & \text { VStr during }\end{array}$ presentation of conditioned reward stimuli in bipolar

4 patients (A) and matched healthy controls (B). Significantly reduced reward signals in the $v$ Str of bipolar patients (C; patients<controls). D-E: Reduced

2 functional coupling between anteroventral prefrontal cortex (avPFC) and $v$ Str in bipolar patients compared to healthy controls ( $D$; patients<controls; $p<0.05$, FDR-corrected for multiple comparisons across the entire brain), and decreased top-down regulation of reward signals in the $v$ Str of bipolar patients $(E$; patients<controls; $p<0.05$, FWEcorrected for small volume, $6 \mathrm{~mm}$ box at $1212-3$, a priori coordinates from Diekhof \& Gruber, 2010). Tvalues are indicated by color bars. For more details see Tables 2 and 3 .

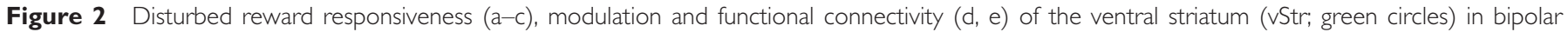
disorder. (a-c) Bottom-up activation of the vStr during presentation of conditioned reward stimuli in bipolar patients (a) and matched healthy controls (b). Significantly reduced reward signals in the vStr of bipolar patients (c; patients<controls). (d, e) Reduced functional coupling between anteroventral

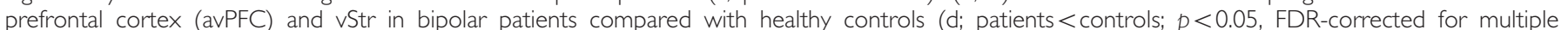

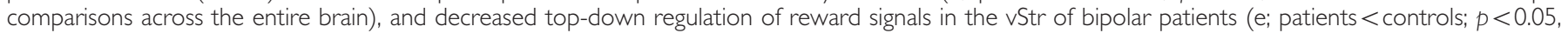

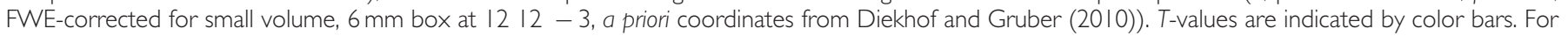
more details, see Tables 2 and 3.

\section{DISCUSSION}

The present study investigated reward-related brain activations and the neurofunctional mechanisms underlying the ability to resist immediate reward desiring in favor of a superordinate goal in bipolar patients using fMRI. Our main findings were a significantly reduced neural response of the vStr to conditioned reward stimuli, an impaired top-down regulation of reward-related activity in the vStr, and a disturbed functional connectivity between the anteroventral prefrontal cortex and the nucleus accumbens in bipolar patients.

There are inconsistent results about reward processing in BD. A recent fMRI study found elevated ventral striatal activity in response to reward stimuli in hypomanic individuals (O’Sullivan et al, 2011), while manic bipolar patients showed high ventral striatal fMRI signals in response to rewarding, but also to non-rewarding stimuli (Abler et al, 2008). This was interpreted as a deficit in distinguishing salient from irrelevant stimuli during mania. With regard to euthymia, elevated ventral striatal activity during reward anticipation, but not outcome, has been reported in bipolar I disorder (Nusslock et al, 2012). Others, however, found that ventral striatal activity during reward anticipation was only elevated in bipolar II patients as compared with both bipolar I patients and healthy controls (Caseras et al, 2013). Conversely, during positive outcome, the bipolar I patients showed greater ventral striatal activity than bipolar II patients in the latter study. The bipolar patients in the present study, however, exhibited a decreased bottom-up response to conditioned reward stimuli in the vStr. Of note, the majority of the abovementioned studies used a monetary incentive delay task similar to Knutson et al (2001) differentiating between reward anticipation and outcome. In contrast, the participants of the present study had been conditioned to associate specific stimuli with a rewarding outcome. According to this, participants showed an immediate, automated bottomup response to the conditioned stimuli. There was no separate anticipation or expectation phase in the present task. Considering these multiple methodological differences in the experimental design, different functional aspects of reward processing probably have been investigated by these different paradigms. This may play a role with respect to our finding of reduced ventral striatal activity in response to conditioned reward stimuli in the bipolar group. 
Furthermore, this reduced ventral striatal activation may be related to the predominant current psychopathological status of the bipolar patients included in our sample (see Table 1), ie, to (sub)depressive symptoms such as anhedonia. Supporting this interpretation, reduced fMRI responses to gains in the vStr have been shown in depressive patients (Forbes et al, 2009; Pizzagalli et al, 2009; Robinson et al, 2012), and ventral striatal activity has been negatively correlated with anhedonia (Keedwell et al, 2005).

Likewise at the behavioral level, we found reduced reward exploitation rates in the bipolar group corresponding to recent reports of dysfunctional reward learning in bipolar patients, which was also correlated with anhedonia (Pizzagalli et al, 2008).

Furthermore, the bipolar patients showed significantly reduced task-related activation in the right middle frontal gyrus (Table 2). This brain region has been shown to be involved in visual working memory for colors and objects by several fMRI studies (Belger et al, 1998; Gruber and von Cramon, 2001; Mohr et al, 2006; Sala et al, 2003). Since memorizing two target colors at the beginning of each block was essential for successful task performance in the present paradigm, reduced activation in this region in bipolar patients, which was significantly correlated with their reduced performance rates in correct target acceptance, could indicate a disturbance of such working memory processes. These findings are in line with reports of visuospatial and working memory deficits in BD (Frantom et al, 2008; Lera-Miguel et al, 2011; Pan et al, 2011).

More importantly, in the DRD suppression of rewardrelated activation was significantly reduced in the bipolar patients, most prominently in the bilateral vStr $(p<0.05$, FWE-corrected for small volume). We attribute this finding to an impaired top-down control of prefrontal regions on the vStr. Our novel finding of disturbed avPFC-vStr functional connectivity strongly corroborates this interpretation. While the healthy controls exhibited a negative functional interaction between the vStr and the avPFC as demonstrated by several earlier studies using the DRD paradigm (Diekhof and Gruber, 2010), this negative functional coupling was severely impaired in the bipolar patients. These results are indicative for a disturbed topdown control of reward signals in the mesolimbic reward system by the avPFC in BD. Disturbed functional connectivity patterns may be related to abnormalities within white-matter tracts connecting the frontal cortex with temporal and parietal cortices and the fronto-subcortical and fronto-limbic circuits, as it has been shown by several studies in bipolar patients (Brambilla et al, 2009; Heng et al, 2010; Strakowski et al, 2012). In particular, the impairment of connectivity between avPFC and vStr fits in the suggested prefrontal-striatal-pallidal-thalamic network dysfunction probably underlying BD (Strakowski et al, 2012).

In correspondence to the fMRI results, we found elevated impulsivity scores and significantly reduced task performance rates in the bipolar patients, particularly in the DRD. These findings further support our interpretation of impaired top-down control mechanisms clinically and behaviorally expressed by enhanced trait impulsivity and reduced DRD performance rates.

We also found an abnormal modulation of brain activity in the triangular part of the left inferior frontal cortex. This brain region has been found to be involved in response inhibition and instrumental learning in go/no-go tasks (Guitart-Masip et al, 2012). Furthermore, brain activity in the inferior frontal gyrus was shown to modulate impulsivity in healthy subjects, with greater impulsivity associated with attenuated inferior frontal gyrus activity (Horn et al, 2003; Townsend et al, 2012). Recently, Townsend et al (2012) reported reduced bilateral inferior frontal gyrus (pars triangularis) activation in a go/no-go tasks in a group of 32 euthymic bipolar patients as a possible trait abnormality. In accordance with this finding, the bipolar patients in the present study showed reduced modulation of brain activity in the inferior frontal gyrus in a situation, where inhibition of a previously conditioned response bias toward immediate reward was required. Beta values extracted from the inferior frontal gyrus were negatively correlated with BIS-11 total scores in the bipolar group providing further support for an association of inferior frontal dysfunction with enhanced impulsivity in BD (see Table 1).

In line with these fMRI findings in the DRD situation, the bipolar patients also showed reduced performance rates in correctly rejecting a conditioned reward stimulus. These results correspond to previous findings of bipolar patients failing to flexibly adapt to change stimulus-reward contingencies and showing deficient response reversal after initial learning (Dickstein et al, 2010; Gorrindo et al, 2005; Johnson et al, 2012b). Another reason for reduced action control in the DRD may be enhanced impulsivity, which is a characteristic feature of $\mathrm{BD}$. Particularly rapid-response impulsivity and a reduced ability to delay response for a reward have been reported in bipolar patients, regardless of mood state or treatment (Strakowski et al, 2010; Swann, 2010; Swann et al, 2009). In line with this, the present bipolar sample showed elevated impulsivity scores (see Table 1).

One limitation of the present study is that almost all bipolar patients included were medicated. There have been findings of decreased differential reward-related brain activation in the vStr and other reward associated areas by a single dose of olanzapine in healthy controls (Abler et al, 2007). On the other hand, schizophrenic patients medicated with first- or second-generation antipsychotics showed normal patterns of VTA and ventral striatal activation during monetary incentive tasks in two independent studies (Abler et al, 2008; Juckel et al, 2006). Recently, a comprehensive review on effects of psychotropic medication on neuroimaging findings in BD showed only limited impact on fMRI results, with predominantly normalizing effects (Hafeman et al, 2012). In the present study, we found no effects of antidopaminergic medication on reward-related striatal activation in the DC, but the extent of suppression of striatal activation in the RC compared with the DC was positively correlated with $\mathrm{CPZ}$ daily dosage. This means that higher $\mathrm{CPZ}$ doses were associated with better (ie, normal) suppression of reward-related activity in the DRD. Thus, in line with the recent review by Hafeman et al (2012) this finding indicates that well-medicated bipolar patients showed normalized top-down suppression of reward signals in the RC (rather than any disturbance induced by the antidopaminergic medication). 
Another limiting aspect of the present study is the study sample size $(n=16)$. However, this sample size is comparable to the number of patients investigated in previously published studies providing significant results, such as Abler et al (2008) $(n=12)$, O'Sullivan et al (2011) $(n=12)$, Caseras et al (2013) $(n=17 / n=15)$ or Nusslock et al (2012) $(n=21)$.

To conclude, the present study provides evidence for a reduced bottom-up responsiveness of the mesolimbic reward system in $\mathrm{BD}$. This finding may be related to residual or state-dependent anhedonic symptoms, as the patients investigated in the present study were euthymic or suffering from mild depressive symptoms. Most importantly, the study gives first direct evidence for an impaired top-down control of the mesolimbic reward system by prefrontal brain regions in $\mathrm{BD}$, possibly underlying the disease-related enhanced trait impulsivity.

\section{FUNDING AND DISCLOSURE}

This work was partially funded by the Deutsche Forschungsgemeinschaft (DFG) via the Clinical Research Group 241 'Genotype-phenotype relationships and neurobiology of the longitudinal course of psychosis', TP 2 (http://www.kfo241.de; grant number GR 1950/5-1). Peter Falkai was honorary speaker for the following companies: Astra Zeneca, Janssen Cilag, Lilly, and Lundbeck. He was member of the Scientific Advisory Boards of Astra Zeneca, Janssen-Cilag, Lilly, and Lundbeck from 2008 to 2010 and acted as a principal investigator in a clinical trial supported by Astra Zeneca from 2006 to 2010. Oliver Gruber was honorary speaker for the following companies: Astra Zeneca, Bristol Myers Squibb, Janssen Cilag, Lilly, Servier, and Otsuka. He has been invited to scientific congresses by Astra Zeneca, Janssen Cilag and Pfizer and has received a research grant from Servier. Dres. Falkai and Gruber report these potential conflicts have no relation to the subject of the present study. The remaining authors declare that, except for income received from their primary employer, no financial support or compensation has been received from any individual or corporate entity over the past three years for research or professional service and there are no personal financial holdings that could be perceived as constituting a potential conflict of interest.

\section{REFERENCES}

Abler B, Erk S, Walter H (2007). Human reward system activation is modulated by a single dose of olanzapine in healthy subjects in an event-related, double-blind, placebo-controlled fMRI study. Psychopharmacology (Berl) 191: 823-833.

Abler B, Greenhouse I, Ongur D, Walter H, Heckers S (2008). Abnormal reward system activation in mania. Neuropsychopharmacology 33: 2217-2227.

Alloy LB, Bender RE, Whitehouse WG, Wagner CA, Liu RT, Grant DA et al (2012). High Behavioral Approach System (BAS) sensitivity, reward responsiveness, and goal-striving predict first onset of bipolar spectrum disorders: A prospective behavioral high-risk design. J Abnorm Psychol 121: 339-351.

Belger A, Puce A, Krystal JH, Gore JC, Goldman-Rakic P, McCarthy G (1998). Dissociation of mnemonic and perceptual processes during spatial and nonspatial working memory using fMRI. Hum Brain Mapp 6: 14-32.
Bermpohl F, Kahnt T, Dalanay U, Hägele C, Sajonz B, Wegner T et al (2010). Altered representation of expected value in the orbitofrontal cortex in mania. Hum Brain Mapp 31: 958-969.

Brambilla P, Bellani M, Yeh P-H, Soares JC, Tansella M (2009). White matter connectivity in bipolar disorder. Int Rev Psychiatry 21: 380-386.

Caseras X, Lawrence NS, Murphy K, Wise RG, Phillips ML (2013). Ventral striatum activity in response to reward: differences between bipolar I and II disorders. Am J Psychiatry 170: 533-541.

Dickstein DP, Finger EC, Skup M, Pine DS, Blair JR, Leibenluft E (2010). Altered neural function in pediatric bipolar disorder during reversal learning. Bipolar Disord 12: 707-719.

Diekhof EK, Falkai P, Gruber O (2008). Functional neuroimaging of reward processing and decision-making: a review of aberrant motivational and affective processing in addiction and mood disorders. Brain Res Rev 59: 164-184.

Diekhof EK, Gruber O (2010). When desire collides with reason: functional interactions between anteroventral prefrontal cortex and nucleus accumbens underlie the human ability to resist impulsive desires. J Neurosci 30: 1488-1493.

Diekhof EK, Keil M, Obst KU, Henseler I, Dechent P, Falkai P et al (2012a). A functional neuroimaging study assessing gender differences in the neural mechanisms underlying the ability to resist impulsive desires. Brain Res 1473: 63-77.

Diekhof EK, Nerenberg L, Falkai P, Dechent P, Baudewig J, Gruber O (2012b). Impulsive personality and the ability to resist immediate reward: an fMRI study examining interindividual differences in the neural mechanisms underlying self-control. Hum Brain Mapp 33: 2768-2784.

Forbes EE, Hariri AR, Martin SL, Silk JS, Moyles DL, Fisher PM et al (2009). Altered striatal activation predicting real-world positive affect in adolescent major depressive disorder. Am J Psychiatry 166: 64-73.

Frantom LV, Allen DN, Cross CL (2008). Neurocognitive endophenotypes for bipolar disorder. Bipolar Disord 10: 387-399.

Friston KJ, Buechel C, Fink GR, Morris J, Rolls E, Dolan RJ (1997). Psychophysiological and modulatory interactions in neuroimaging. Neuroimage 6: 218-229.

Gorrindo T, Blair RJR, Budhani S, Dickstein DP, Pine DS, Leibenluft E (2005). Deficits on a probabilistic response-reversal task in patients with pediatric bipolar disorder. Am J Psychiatry 162: 1975-1977.

Gruber J (2011). A review and synthesis of positive emotion and reward disturbance in bipolar disorder. Clin Psychol Psychother 18: 356-365.

Gruber O, von Cramon DY (2001). Domain-specific distribution of working memory processes along human prefrontal and parietal cortices: a functional magnetic resonance imaging study. Neurosci Lett 297: 29-32.

Guitart-Masip M, Huys QJM, Fuentemilla L, Dayan P, Duzel E, Dolan RJ (2012). Go and no-go learning in reward and punishment: Interactions between affect and effect. Neuroimage 62: 154-166.

Hafeman DM, Chang KD, Garrett AS, Sanders EM, Phillips ML (2012). Effects of medication on neuroimaging findings in bipolar disorder: an updated review. Bipolar Disord 14: 375-410.

Hasler G (2006). Evaluating endophenotypes for psychiatric disorders. Rev Bras Psiquiatr 28: 91-92.

Heng S, Song AW, Sim K (2010). White matter abnormalities in bipolar disorder: insights from diffusion tensor imaging studies. J Neural Transm 117: 639-654.

Horn NR, Dolan M, Elliott R, Deakin JFW, Woodruff PWR (2003). Response inhibition and impulsivity: an fMRI study. Neuropsychologia 41: 1959-1966.

Johnson SL, Carver CS, Gotlib IH (2012a). Elevated ambitions for fame among persons diagnosed with bipolar I disorder. J Abnorm Psychol 121: 602-609. 
Johnson SL, Edge MD, Holmes MK, Carver CS (2012b). The behavioral activation system and mania. Annu Rev Clin Psychol 8: 243-267.

Juckel G, Schlagenhauf F, Koslowski M, Filonov D, Wüstenberg T, Villringer A et al (2006). Dysfunction of ventral striatal reward prediction in schizophrenic patients treated with typical, not atypical, neuroleptics. Psychopharmacology (Berl) 187: 222-228.

Keedwell PA, Andrew C, Williams SCR, Brammer MJ, Phillips ML (2005). The neural correlates of anhedonia in major depressive disorder. Biol Psychiatry 58: 843-853.

Knutson B, Fong GW, Adams CM, Varner JL, Hommer D (2001). Dissociation of reward anticipation and outcome with eventrelated fMRI. Neuroreport 12: 3683-3687.

Lera-Miguel S, Andrés-Perpiñá S, Calvo R, Fatjó-Vilas $M$, Fañanás L, Lourdes F et al (2011). Early-onset bipolar disorder: how about visual-spatial skills and executive functions? Eur Arch Psychiatry Clin Neurosci 261: 195-203.

Linke J, King AV, Rietschel M, Strohmaier J, Hennerici M, Gass A et al (2012). Increased medial orbitofrontal and amygdala activation: evidence for a systems-level endophenotype of bipolar I disorder. Am J Psychiatry 169: 316-325.

Liu X, Hairston J, Schrier M, Fan J (2011). Common and distinct networks underlying reward valence and processing stages: a meta-analysis of functional neuroimaging studies. Neurosci Biobehav Rev 35: 1219-1236.

McClure SM, York MK, Montague PR (2004). The neural substrates of reward processing in humans: the modern role of FMRI. Neuroscientist 10: 260-268.

McDonald C, Bullmore ET, Sham PC, Chitnis X, Wickham H, Bramon $\mathrm{E}$ et al (2004). Association of genetic risks for schizophrenia and bipolar disorder with specific and generic brain structural endophenotypes. Arch Gen Psychiatry 61: 974-984.

Mohr HM, Goebel R, Linden DEJ (2006). Content- and task-specific dissociations of frontal activity during maintenance and manipulation in visual working memory. J Neurosci 26: 4465-4471.

Nusslock R, Almeida JR, Forbes EE, Versace A, Frank E, Labarbara EJ et al (2012). Waiting to win: elevated striatal and orbitofrontal cortical activity during reward anticipation in euthymic bipolar disorder adults. Bipolar Disord 14: 249-260.

O'Doherty JP (2004). Reward representations and reward-related learning in the human brain: insights from neuroimaging. Curr Opin Neurobiol 14: 769-776.
O’Sullivan N, Szczepanowski R, El-Deredy W, Mason L, Bentall RP (2011). fMRI evidence of a relationship between hypomania and both increased goal-sensitivity and positive outcome-expectancy bias. Neuropsychologia 49: 2825-2835.

Pan Y-J, Hsieh MH, Liu S-K (2011). Visuospatial working memory deficits in remitted patients with bipolar disorder: susceptibility to the effects of GABAergic agonists. Bipolar Disord 13: 365-376.

Pizzagalli DA, Goetz E, Ostacher M, Iosifescu DV, Perlis RH (2008). Euthymic patients with bipolar disorder show decreased reward learning in a probabilistic reward task. Biol Psychiatry 64: 162-168.

Pizzagalli DA, Holmes AJ, Dillon DG, Goetz EL, Birk JL, Bogdan R et al (2009). Reduced caudate and nucleus accumbens response to rewards in unmedicated subjects with major depressive disorder. Am J Psychiatry 166: 702-710.

Robinson OJ, Cools R, Carlisi CO, Sahakian BJ, Drevets WC (2012). Ventral striatum response during reward and punishment reversal learning in unmedicated major depressive disorder. Am J Psychiatry 169: 152-159.

Sala JB, Rämä P, Courtney SM (2003). Functional topography of a distributed neural system for spatial and nonspatial information maintenance in working memory. Neuropsychologia 41: $341-356$

Strakowski SM, Adler CM, Almeida J, Altshuler LL, Blumberg HP, Chang KD et al (2012). The functional neuroanatomy of bipolar disorder: a consensus model. Bipolar Disord 14: 313-325.

Strakowski SM, Fleck DE, DelBello MP, Adler CM, Shear PK, Kotwal $\mathrm{R}$ et al (2010). Impulsivity across the course of bipolar disorder. Bipolar Disord 12: 285-297.

Swann AC (2010). Mechanisms of impulsivity in bipolar disorder and related illness. Epidemiol Psichiatr Soc 19: 120-130.

Swann AC, Lijffijt M, Lane SD, Steinberg JL, Moeller FG (2009). Increased trait-like impulsivity and course of illness in bipolar disorder. Bipolar Disord 11: 280-288.

Townsend JD, Bookheimer SY, Foland-Ross LC, Moody TD, Eisenberger NI, Fischer JS et al (2012). Deficits in inferior frontal cortex activation in euthymic bipolar disorder patients during a response inhibition task. Bipolar Disord 14: 442-450.

Williams JR (2008). The Declaration of Helsinki and public health. Bull World Health Organ 86: 650-652.

Supplementary Information accompanies the paper on the Neuropsychopharmacology website (http://www.nature.com/npp) 